Transitional discussions to be made a regular agenda item at team meetings.

Care co-ordinator to be informed and reminded that transitional plans need to be explored with young people.

Following a re-audit of this data 6 months on $100 \%$ of cases over the age of 18 were closed and transition was discussed in the remaining $56 \%$.

\section{Where is my sample? Investigating pre-analytical pathology sampling errors in a psychiatric hospital}

\author{
Emma Scholes-Pearson ${ }^{1 \star}$ and Nieves Mercadillo ${ }^{2}$ \\ ${ }^{1}$ Warrington and Halton Teaching hospitals and ${ }^{2}$ North West \\ Boroughs Healthcare NHS Foundation Trust \\ ${ }^{*}$ Corresponding author.
}

doi: 10.1192/bjo.2021.911

Aims. Errors in the pathology sampling process can be costly for all stakeholders in any clinical setting; however, this process is often overlooked within psychiatry. Over the space of just a few short months at Hollins Park Psychiatric Hospital (HPH) such errors were reported to be numerous with staff raising multiple issues relating to the pathology sampling process. These issues often had a negative impact on patient care, leading to outcomes such as as re-bleeding of patients and delays in interventions. Here, we aim to identify the predominant sources of error in this process and suggest possible improvements to minimise these errors in the future. Method. Initially, we mapped and analysed each step of the sampling process as it is currently performed in order to identify areas of possible improvement. We then distributed questionnaires to all junior doctors - who are responsible for the handling of samples within the clinical setting - in order to establish error type and frequency. Questions also assessed individual confidence and familiarity with the sampling process.

Result. When mapping the sampling process, we identified all key steps required when sending samples from $\mathrm{HPH}$ to Warrington and Halton Hospitals laboratory. This included one pathway for sending routine bloods, and one pathway for urgent bloods. The process for sending routine bloods required more steps and ultimately took longer for samples to reach the laboratory - as expected. Of the issues identified during mapping of the pre-analytical phase, a majority of $77.7 \%$ of clinicians reported samples had gone missing or were unreported - with the reasons for this being undetermined in most cases - and 55.5\% reported their samples never reached the lab. While on the whole participants were comfortable with the steps involved in sending samples to the laboratory, $77.7 \%$ were not aware of the requirement to log samples as they were being sent. Conclusion. The reasons underlying errors in the sampling process at HPH were multifactorial and included a lack of clinician familiarity with correct procedure, poor sample recording/tracking and lengthy transit times between the patient and laboratory. Here we outline some simple evidence-based recommendations (including education of staff and improved tracking through an electronic requesting system) to help reduce errors and streamline the sampling process in the hopes of improving both efficiency and accuracy, reducing the financial and clinical impact.

\section{High dose antipsychotic treatment monitoring audit}

Jake Scott ${ }^{\star}$ and Jose Belda

Sussex Partnership NHS Foundation Trust

${ }^{\star}$ Corresponding author.

doi: 10.1192/bjo.2021.912
Aims. To quantify how many patients were prescribed high dose antipsychotic treatment (HDAT) and establish whether guidance for monitoring HDAT was being followed in an Assertive Outreach Team.

Background. Severe mental health disorders are associated with significant premature mortality, predominantly due to physical health conditions. Antipsychotic medications are associated with side effects, including metabolic syndrome and QT prolongation, which increase the risk of serious physical illness. HDAT is defined as when the total dose of antipsychotics prescribed exceeds $100 \%$ of the maximum BNF dose, if each dose is expressed a percentage of its maximum dose. There is limited evidence of clinical benefit with HDAT but an increased risk of side effects. Patients prescribed HDAT should therefore be monitored for side effects and clinical benefit. Sussex Partnership NHS Foundation Trust developed a form specifically for this purpose, to be completed in addition to a physical health assessment.

Method. All patients on caseload were audited using the electronic notes. Current inpatients were excluded, as inpatient HDAT monitoring forms are attached to paper drug charts and therefore were not available for review.

Result. A total of 61 patients were audited. Nine were excluded due to being inpatients. 16 were on community treatment orders and 26 were prescribed a long-acting antipsychotic injection. 10 were prescribed clozapine. The median number of medications prescribed was one. Four patients were prescribed HDAT ranging from $117-150 \%$ of the maximum BNF dose. Of these four, one had a HDAT form but this was out of date. 39 of 52 (75\%) patients audited had had a physical health assessment in the past 12 months. Two of the 13 missing a physical health assessment were on HDAT.

Conclusion. Physical health monitoring should be carried out for all patients on antipsychotics, but is particularly important for patients on HDAT. This audit identified a problem in both general physical health checks and HDAT monitoring. On discussion with the multi-disciplinary team a number of barriers to appropriate physical health monitoring were identified. There was a lack of awareness within the multi-disciplinary team that patients were receiving HDAT and regarding the implications for side effects. A reliable system to highlight the need for physical health checks was also missing and the team did not have sufficient equipment to perform the necessary checks. Identifying these barriers should enable improvements in physical health and HDAT monitoring which can be re-audited.

\section{Psychiatric liaison team memory pathway: does it achieve the standards set out in NICE clinical guideline 97 ?}

Manouri Senaratne ${ }^{1 \star}$, Afshan Jabeen ${ }^{1}$, Alisha Hyams ${ }^{1}$, Carol Deary ${ }^{1}$ and Sarah Stathers ${ }^{2}$

${ }^{1}$ South West Yorkshire Partnership NHS Foundation Trust and

${ }^{2}$ South West Yorkshire Partnership NHS Foundation Trust; Cumbria, Northumbria and Tees Valley NHS Foundation Trust ${ }^{\star}$ Corresponding author.

doi: 10.1192/bjo.2021.913

Aims. Early assessment, diagnosis and management for people living with dementia is essential, both for the patient and their carers. We recognised delays in established local pathways when patients had unplanned acute hospital admissions preventing them from attending memory diagnostic appointments. The Psychiatric Liaison Team (PLT) Memory Pathway was introduced 